$17^{\text {th }}$ European Symposium on Computer Aided Process Engineesing - ESCAPE17

V. Plesu and P.S. Agachi (Editors)

(C) 2007 Elsevier B.V./Ltd. All rights reserved.

\title{
Methodology for the optimal thermo-economic, multi-objective design of thermochemical fuel production from biomass
}

\author{
Martin Gassner, François Maréchal
}

Laboratory for Industrial Energy Systems, Ecole Polytechnique Fédérale de Lausanne, CH-1015 Lausanne, Switzerland,francois.maréchal@epfl.ch

\begin{abstract}
This paper addresses a methodology for the optimal conceptual design of thermochemical fuel production processes from biomass. A decomposed modelling approach with separate energy-flow, energy-integration and economic models is presented and coupled to multi-objective optimisation, which allows to generate a set of optimal process flowsheets that constitute a sound basis for the synthesis of a viable process.
\end{abstract}

\section{Keywords}

Biofuels, process design, thermo-economic modelling, optimisation.

\section{Introduction}

Biofuel production processes are highly integrated energy conversion processes whose design has an important impact on the performance of the overall system. In addition to the technology development, the efficiency of such processes relies on the quality of the design and mainly on the quality of the process integration. Systematic methodologies for preliminary process design based on process integration techniques and multi-objective optimisation have been developed and applied to power plant and solid oxide fuel cell system design [1, 2]. In the field of biofuel production, such computer aided process synthesis methodologies have not really been applied. Most of the thermo-economic process investigations addressing the production of Fischer Tropsch (FT) liquids, synthetic natural gas (SNG) and the coproduction of these fuels are 
based on conventional simulation of some flowsheet scenarios developed by hand $[3,4]$.

The present paper aims at presenting a process design methodology to be applied for the conceptual design of thermochemical biofuel production processes and demonstrate it by the example of SNG production from wood.

\section{Design methodology}

The basic concept of our method for optimal thermo-economic, multi-objective design is the decomposition of the problem into several parts, as illustrated in figure 1. After identifying suitable technology for the conversion steps, energyflow, energy-integration and economic models of the equipment and their interactions are integrated in a multi-objective optimisation framework to compute a set of optimal process configurations with respect to different design objectives. An analysis of the optimisation results with regard to multiple criteria then results in the synthesis of a sound conceptual plant flowsheet.

\subsection{Block flow superstructure}

In the first step of the design, the product specifications and the available raw materials and energy resources are investigated and the general requirements on the process are defined, which determines feasible production pathways, required process steps and intermediate products. Suitable technologies for the main conversion routes and auxiliary operations such as feed preparation and stream conditioning are identified and assembled in a process block flow superstructure. An example for the wood to SNG process is given on figure 2. The definition of possible material pathways and the identification of the range of operating conditions for which the transformations are thermodynamically and technically feasible concludes the technology identification step and results in the proper definition of the design problem.

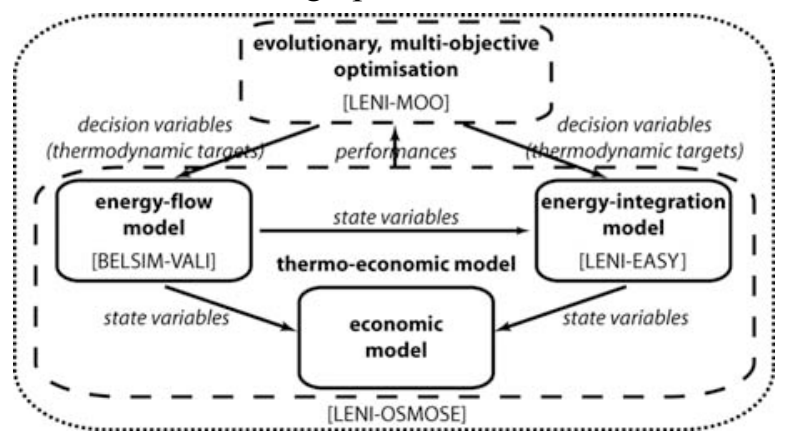

Figure 1: Design methodology overview. 
Methodology for the optimal thermo-economic, multi-objective design of thermochemical fuel production from biomass

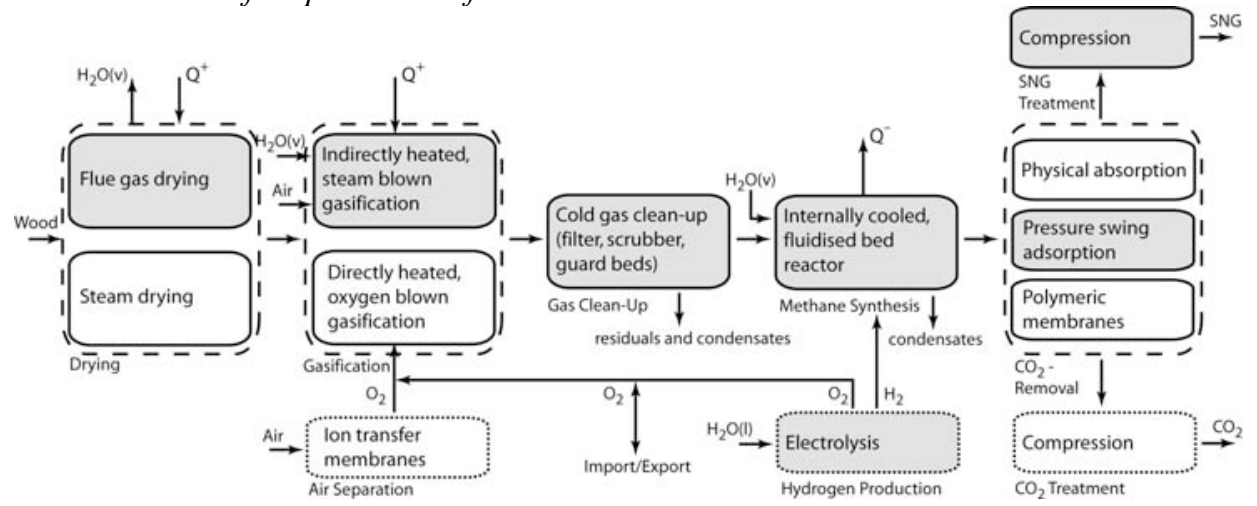

Figure 2: Process superstructure. Dashed boxes assemble competing technologies and dotted ones are used for optional equipment. The examined process configuration is shown shaded.

\subsection{Thermo-economic model development}

\subsubsection{Flowsheet generation}

One of the key advantages of the design approach presented in this paper is to systematically generate the process flowsheet in two successive steps. The operation of the process units is calculated in the energy-flow model, followed by determining the material and energy flows by the heat and power integration. Unlike conventional flowsheeting methods, the topology of the heat exchanger network and the fuel supply are not defined a priori, but computed in the integration step, which makes the method very suitable for preliminary process design.

Energy-flow model. For all process equipments of the block flow superstructure, an energy-flow model of each thermodynamic transformation is developed [5]. These models satisfy the mass and energy balances and link the inlet and outlet streams by model equations representing the physical and chemical conversion of species. The thermodynamic state of the streams and the heat and power requirements of the transformation are calculated and allow to determine the list of the hot and cold streams to be considered in the energy integration.

Energy-integration model. Once the heat and power requirements of the transformations are defined, the heat cascade is used to model the heat exchanger network. Flows in the system are optimised in order to maximise the combined heat and power production in the plant. The thermal effects of each sequence of operations without stream bifurcation are grouped and constitute the units whose flowrates are to be computed in the integration problem. In order to supply the energy requirement above the pinch, combustion of fuels available on-site is considered. Dissociating the effects of the fuel and 
combustion air as outlined in [6], the thermal effects of usable waste and retentate streams are formulated. In the example presented here, waste and process streams may be used as fuel to close the balance leading to a reduction of the flows in the main conversion route. The choice of using optional energy conversion and recovery equipment like heat pumps, gas turbines and Rankine cycles is formulated by means of binary variables. The structure and operating conditions of these units are predefined and considered as decision variables of the overall design problem. If a certain technology is considered, only the corresponding flowrates are calculated by the energy-integration model which is solved as a mixed integer linear programming problem that maximises the combined fuel, heat and power production of the process. The integration of process modelling and process integration was also proposed in [7]. However, in their case, the utility streams and the combined heat and power production was not considered and only the energy consumption was targeted.

The example of indirectly heated gasification. Contrary to the conventional simulation approach applied in [8], the energy-flow model only deals with the actual transformation (i.e. the gasification) and determines its heat demand. The technological implementation to satisfy this demand (i.e. combustion of residual char and cold product gas in an attached reactor and heat transfer by recirculation of hot bed material) is not specified, but the heat requirement itself is transferred to the energy-integration model. Instead of fixing one specific stream as fuel for the gasifier, its flow will be determined to satisfy the energy integration of the whole system. In the examined process configuration, unconverted char and gaseous residue of the condensates from methane synthesis are considered as waste streams to be burnt. Additional streams for fuel supply are chosen among dried wood and the hot and cold synthesis gas from the gasifier and gas cleaning outlets instead of only considering the cold synthesis gas as fuel.

\subsubsection{Equipment sizing and cost estimation}

The thermodynamic state of the process streams are specified as decision variables or result from them through the flowsheet generation step. For each process equipment, these conditions represent the design target to be met. A preliminary sizing and cost estimation procedure has been implemented for each unit reflecting the direct influence of the design variables on the investment cost into account. For this purpose, equipment design heuristics from the literature [9] combined with data from existing experimental and pilot plant facilities are used to roughly dimension the major process equipment for a given production scale. The grass roots cost $\mathrm{C}_{\mathrm{GR}}$ of the plant, i.e. the total investment cost for a new facility excluding land, is then estimated following the method of [10]. 
Methodology for the optimal thermo-economic, multi-objective design of thermochemical fuel production from biomass

\subsection{Generation of optimal flowsheets}

In order to identify best feasible solutions preserving the multiple aspects of the design problem, an evolutionary, multi-objective optimisation algorithm using clustering techniques [11] is applied to the thermo-economic process model. This step can be seen as the generation of a set of optimal flowsheets for a specific production setting, i.e. for a given economic environment and available infrastructure of energy services. The optimisation problem is set up by defining two performance indicators as objectives, whereas at least one of them is consistent with the objective used to compute the energy integration. The decision variables cover both the conversion pathway, equipment choices and the process conditions respecting the domain in which the operations are considered to be feasible.

For the design example of this paper, the plant is supposed to be connected to the gas and electricity grids. This allows for gas production and power recovery from excess heat by means of a Rankine cycle, whereas no external heat sources or heat marketing opportunities are available. In the optimisation problem, two objectives have been considered, i.e. the plant's grass roots cost $\mathrm{C}_{\mathrm{GR}}$ and operating cost $\mathrm{C}_{\mathrm{OP}}$ including the expenses for raw materials, utilities, labour and maintenance. The energy integration is accomplished minimising the costs for raw material and utilities.

\subsection{Results analysis and process synthesis}

The optimal trade-off between grass roots and operating cost resulting from optimisation (figure 3 ) represents the set of potentially best process flowsheets and concludes the conceptual process design. A detailed analysis of these numerically generated configurations with regard to multiple criteria will allow to choose the best process design and do the synthesis of a viable process.

\section{Conclusions}

Due to the separate modelling of the thermodynamic conversions and their thermal integration, the methodology presented here avoids to restrict the investigated process layouts to a very limited number of scenarios at an early stage of the design. Instead, its coupling with cost estimation procedures that consider the thermodynamic conditions and the usage of a multi-objective optimisation algorithm allows to systematically generate a set of best flowsheets for a given production setting. The methodology is thus very suitable for the conceptual design of integrated biofuel plants. It should be understood as a tool that efficiently eliminates solutions that are not worth investigating in detail, identifies the most promising process layouts and operating conditions and guides the efforts in R\&D towards potentially optimal plants. 


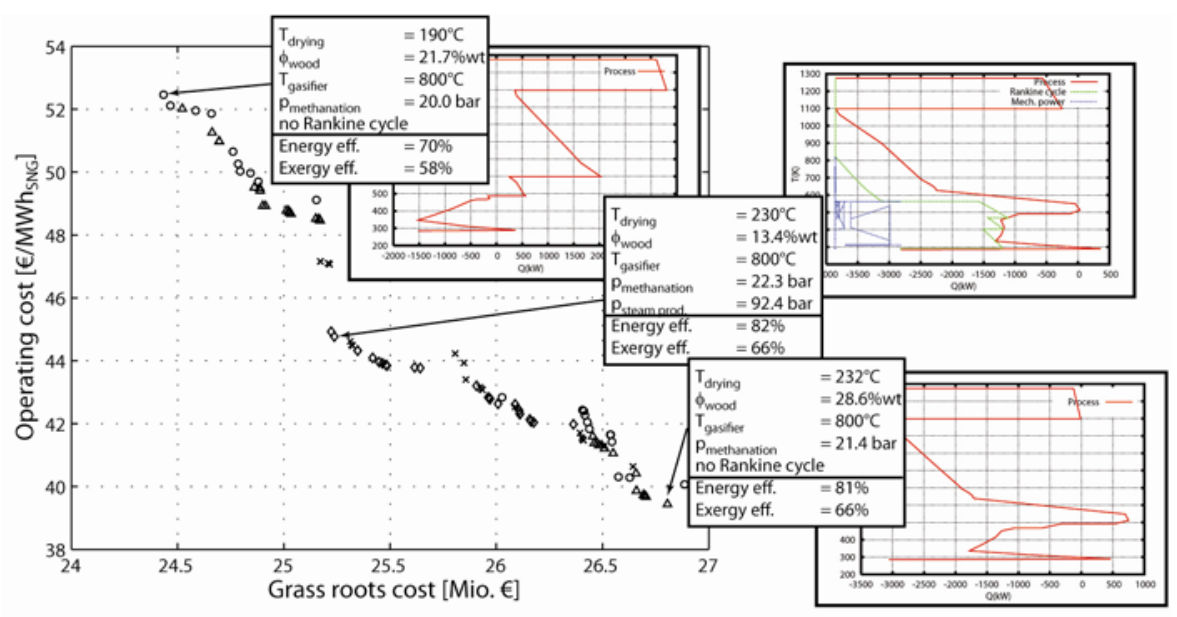

Figure 3: Pareto trade-off between capital and operating costs for four clusters and three exemplary configurations with their composite curves.

\section{References}

1. Bolliger, R., Favrat, D., Maréchal, F., Advanced power plant design methodology using process integration and multi-objective thermo-economic optimisation. Proceedings of ECOS 2005.

2. Palazzi, F., Maréchal, F., van Herle, J., Autissier, N., A methodology for thermo-economic modeling and optimisation of SOFC systems. Chem. Eng. Trans. 7 (2005), 13-18.

3. Mozaffarian, M., Zwart, R., Feasibility of biomass/waste-related SNG production technologies. ECN-C-03-66, Petten, Netherlands, 2003.

4. Zwart, R.W.R., Boerrigter, H., High efficiency co-production of synthetic natural gas (SNG) and Fischer-Tropsch (FT) transportation fuels from biomass. Energy and Fuels 19 (2005), 591-597.

5. Gassner, M., Maréchal, F., Thermo-economic model of a process converting wood to methane. Submitted to Biomass and Bioenergy, 2006.

6. Maréchal, F., Kalitventzeff, B., Process integration: Selection of the optimal utility system. Comp. Chem. Eng. 22 (1998), S149-S156.

7. Hostrup, M., Gani, R., Kravanja, Z., Sorsak, A., Grossmann, I., Integration of thermodynamic insights and MINLP optimization for the synthesis, design and analysis of process flowsheets, Comp. Chem. Eng. 25 (2001), 73-83

8. Schuster, G., Löffler, G., Weigl, K., Hofbauer, H., Biomass steam gasification - an extensive parametric modeling study. Bioresource Technology 77 (2001), 71-79.

9. Ulrich, G.-D., A guide to chemical engineering process design and economics. Wiley, New York, 1984.

10. Turton, R., Bailie, R.C., Whiting, W.B., Shaeiwitz, J.A., Analysis, synthesis, and design of chemical processes. Prentice Hall, New Jersey, 1998.

11. Leyland, G.B., Multi-objective optimisation applied to industrial energy problems. $\mathrm{PhD}$ thesis no. 2572, Swiss Federal Institute of Technology, Lausanne, 2002. 